\title{
Ocriplasmin — variable efficacy?
}

\author{
David H. W. Steel ${ }^{1,2} \cdot$ David Wong $^{3}$
}

Received: 21 May 2016 / Accepted: 25 May 2016/Published online: 4 June 2016

(C) Springer-Verlag Berlin Heidelberg 2016

For most vitreoretinal specialists, the adoption of ocriplasmin (OCP) into their practice remains controversial. This might be due to the varying experience, positive and negative, of individual practitioners. In this issue, in a meta-analysis of over 800 patients from 18 series, Chatziralli et al. showed that the rate of release of vitreomacular adhesion (VMA) was $46 \%$ but with a very wide range (from 0 to $71 \%$ ) and wide standard deviation $(21 \%)$ between series [1]. If you are one of the surgeons getting a success rate of closer to $70 \%$, you might be enthusiastic; alternatively, surgeons getting nearer $0 \%$ are likely to give up using OCP. There needs to be some explanation for this wide variation.

Randomised controlled trials (RCT) give us a high level of evidence to guide us in our clinical practice. Nevertheless, there is a need for real-life evidence. In the past, the results of some major RCTs simply could not be replicated. RCTs have strict inclusion and exclusion criteria and therefore limited external validity. In everyday practice, the selection of cases will be different; it could be broader or more restricted. The mean rate of $46 \%$ in this meta-analysis is better than the landmark studies on OCP published in 2012 [2]. It is already known that phakic patients, female gender, absence of epiretinal membrane (ERM), and adhesions less than $1500 \mu \mathrm{m}$ have

David H. W. Steel

David.steel@ncl.ac.uk

1 Sunderland Eye Infirmary, Queen Alexandra Road, Sunderland, UK

2 Institute of Genetic Medicine, Newcastle University, Newcastle Upon Tyne, UK

3 St. Paul's Eye Unit, Royal Liverpool University Hospital Trust, Liverpool, UK a higher rate of release of VMA [2, 3]. Could the wide variation in efficacy in the 18 series reviewed by Chatziralli et al. be simply a reflection of the differences in selection between series? Indeed, the VR interface in VMA is very variable, not just by simplistic measures of extent. It is noteworthy that in the phase 3 trials the presence of ERM anywhere on the OCT, not merely at the point of VR attachment, was marked as present. Hyperreflectivity of the zone of VR interface was not scored, and is a known factor in VR release [4]. Furthermore, it is known that several diseases are associated with increased expression of adhesion molecules at the VR interface, in the absence of discernible ERM explaining the reduced effect of OCP in patients with concomitant disease such as DMO [5].

In addition to selection, another possible reason for the variation in efficacy is the practicalities of the use of the drug in the clinic. OCP is stored at $-25^{\circ} \mathrm{C}$ in a $\mathrm{pH}$ of 3.1 , which prevents serine de-protonation and limits the interaction of the drug with its substrates. Hence the important recommendation that non-buffered saline ( $\mathrm{pH}$ 5.5) should be used to reconstitute it, prolonging its action prior to injection into the [neutral $\mathrm{pH}$ ] eye. Once defrosted and reconstituted, autolysis begins and unless the drug is used immediately, its effectiveness might be affected. Once injected it continues to autolyse, and also interacts with other substrates in the vitreous cavity. This process is in turn limited by the action of naturally occurring serine protease inhibitors whose levels vary by disease [6]. To perform its desired action of VR release from the initial injection point, it has to diffuse to the area of VR adhesion. With second-order pharmacokinetics the distance and rate of the required diffusion to the VR attachment point are critical, and it can be seen that a range of factors will affect the dose reaching the point of VR adhesion, including the depth of injection and the structure of the vitreous. This will not only 
affect the efficacy but also potentially the rate of off-target side-effects in the outer retina and zonules.

Another justification for real-life studies is that more cases are needed to capture complications that occur infrequently or only with longer follow-up. The case of cox-2 inhibitor rofecoxib (Vioxx), which was introduced in 1999 but was subsequently withdrawn in 2004, when it was confirmed that the drug was associated with $40 \%$ increase in cardiovascular event or death, is well known [7]. Chatziralli et al. used the reported complications not just from the series included in the meta-analysis, but included all 194 papers which totalled 874 eyes. This publication highlights the value of meta-analysis, as some complications were not identified in the original MIVITRUST study. These include changes to the ellipsoid zone, electroretinographic changes, and enlargement of the macular hole in failed cases. It is at least reassuring that most of the adverse effects reported have been reversible and transient, although further study is needed.

If OCP is to gain widespread acceptance, there has to be more to recommend it other than its safety profile. There are other options for treating symptomatic VMT and macular holes. The drug is expensive. Chatziralli et al. included in their meta-analysis randomised controlled trials, case-controlled studies, and case series. The heterogeneity of the series, the lack of control groups, and especially the differences in selection criteria make it difficult to compare. The authors seem to suggest that selection is the key. In this regard, we would like to highlight a recent publication by Steel et al. By selecting using OCT parameters (the difference between base diameter and the minimal linear diameter), the group showed that it was possible to predict closure of macular hole with high confidence [8]. Ultimately, it is a higher degree of certainty of success that will persuade many surgeons.

\section{Compliance with ethical standards}

Financial disclosures David Steel declares that he has attended advisory boards for Alcon in connection with ocriplasmin.

\section{References}

1. Chatziralli I, Theodossiadis G, Xanthopoulou P, Miligkos M, Sivaprasad S, Theodossiadis P (2016) Ocriplasmin use for vitreomacular traction and macular hole: a meta-analysis and comprehensive review on predictive factors for vitreous release and potential complications. Graefes Arch Clin Exp Ophthalmol [Epub ahead of print]

2. Stalmans P, Benz MS, Gandorfer A, MIVI-TRUST Study Group et al (2012) Enzymatic vitreolysis with ocriplasmin for vitreomacular traction and macular holes. N Engl J Med 367:606-615

3. Haller JA, Stalmans P, Benz MS, MIVI-TRUST Study Group et al (2015) Efficacy of intravitreal ocriplasmin for treatment of vitreomacular adhesion: subgroup analyses from two randomized trials. Ophthalmology 122:117-122

4. Elbendary AM, Elwan MM, Azzam HA, Eldeeb DR (2011) Predictability of vitreous detachment following intravitreal plasmin injection in diabetic macular edema associated with vitreomacular traction. Curr Eye Res 36:534-539

5. Costa Ede P, Rodrigues EB, Farah ME, Sebag J, Meyer CH (2011) Novel vitreous modulators for pharmacologic vitreolysis in the treatment of diabetic retinopathy. Curr Pharm Biotechnol 12:410-422

6. de Smet MD, Jonckx B, Vanhove M et al (2012) Pharmacokinetics of ocriplasmin in vitreous. Invest Ophthalmol Vis Sci 53:8208-8213

7. h t t p :// w w w f f a . gov/drugs/drugsafety/ postmarketdrugsafetyinformationforpatientsandproviders/ ucm106290.htm. Accessed 19 May 2016

8. Steel DH, Parkes C, Papastavrou VT et al (2016) Predicting macular hole closure with ocriplasmin based on spectral domain optical coherence tomography. Eye (Lond) 30:740-745 